\title{
Cosegregation of the Renin Allele of the Spontaneously Hypertensive Rat with an Increase in Blood Pressure
}

Theodore W. Kurtz, Lizette Simonet, Pokar M. Kabra, Steven Wolfe, Lawrence Chan, and Brian L. Hjelle

Department of Laboratory Medicine, University of California San Francisco, San Francisco, California 94143

\begin{abstract}
The spontaneously hypertensive rat (SHR) exhibits alterations in the renin-angiotensin-aldosterone system which are similar to those that characterize patients with "nonmodulating" hypertension, a common and highly heritable form of essential hypertension. Accordingly, we determined whether the inheritance of a DNA restriction fragment length polymorphism (RFLP) marking the renin gene of the SHR was associated with greater blood pressure than inheritance of a RFLP marking the renin gene of a normotensive control rat. In an F2 population derived from inbred SHR and inbred normotensive Lewis rats, we found the blood pressure in rats that inherited a single SHR renin allele to be significantly greater than that in rats that inherited only the Lewis renin allele. To the extent that the SHR provides a suitable model of "nonmodulating" hypertension, these findings raise the possibility that a structural alteration in the renin gene, or a closely linked gene, may be a pathogenetic determinant of increased blood pressure in one of the most common forms of essential hypertension in humans. (J. Clin. Invest. 1990. 85:1328-1332.) hypertension • genetics $\bullet$ renin
\end{abstract}

\section{Introduction}

The spontaneously hypertensive rat (SHR), ${ }^{1}$ initially bred in Kyoto, Japan, is the most widely studied animal model of human essential hypertension $(1,2)$. This strain exhibits alterations in the renin-angiotensin-aldosterone system $(3,4)$ which are similar to those that characterize patients with

Address reprint requests to Dr. T. W. Kurtz, Department of Laboratory Medicine, University of California San Francisco, Box 0134, San Francisco, CA 94143.

Dr. B. L. Hjelle's present address is the Dept. of Pathology, University of New Mexico School of Medicine, Albuquerque, NM 87131.

Received for publication 31 October 1989 and in revised form 20 December 1989.

1. Abbreviations used in this paper: $\mathrm{LEW}$ rat, Lewis rats; $\mathrm{R}$ rat, Dahl salt-resistant rat; RFLP, restriction fragment length polymorphism; $S$ rat, Dahl salt-sensitive rat; SHR, spontaneously hypertensive rat; VNTR, variation in the number of tandem repeats; WKY rat, WistarKyoto rat.

J. Clin. Invest.

(c) The American Society for Clinical Investigation, Inc. 0021-9738/90/04/1328/05 \$2.00

Volume 85, April 1990, 1328-1332 "nonmodulating" hypertension, a common form of essential hypertension that appears to be highly heritable $(5,6)$. In these patients, changes in sodium chloride intake fail to modulate renal vascular and adrenal responsiveness to intravenously infused angiotensin II. Williams, Hollenberg, and colleagues have suggested that in both "nonmodulators" and SHRs, increased blood pressure may be a consequence, at least in part, of abnormal renal hemodynamics stemming from increased activity of the renin-angiotensin system within the kidney (4, 5 ). The renin-angiotensin-aldosterone axis plays an important role in the regulation of blood pressure and it has long been suspected that this system might participate in the pathogenesis of essential hypertension (7). Accordingly, we determined whether the inheritance of a restriction fragment length polymorphism (RFLP) marking the renin gene of the SHR was associated with greater blood pressure than the inheritance of a RFLP marking the renin gene of a normotensive control rat. In an F2 population derived from inbred SHR and inbred normotensive Lewis rats, we have found the blood pressure in rats inheriting a single SHR renin allele to be significantly greater than that in rats inheriting only the Lewis renin allele.

\section{Methods}

Experimental protocol. Inbred male SHR (SHR/NCrlBR) and female Lewis rats (LEW/NCrlBR, hereafter referred to as LEW rats) were obtained from Charles River Breeding Laboratories, Inc., Wilmington, MA. All rats were housed under identical physical and environmental conditions and were fed Rat Chow (Formula 5008, containing 0.58\% sodium chloride; Ralston Purina Co., Richmond, IN) and tap water ad lib. At the time of these studies, the parental SHR and LEW strains had been maintained by brother-sister mating for over 40 generations and were presumed to be fully inbred. To test this presumption, we performed DNA fingerprint analyses on samples of genomic DNA from SHR and LEW rats which were randomly obtained from Charles River Laboratories, Inc. The parental strains were then crossed to produce F1 rats that were intercrossed to yield an $F 2$ population of 101 rats. At 22-26 wk of age, mean arterial pressures were directly measured in unanesthetized, unrestrained rats from the SHR, LEW, and F2 populations. After completing the blood pressure measurements, spleens were removed for extraction of DNA and renin gene typing. To compare the renin RFLP observed in SHR with that observed in a different animal model of hypertension, we also performed renin gene typing of Dahl salt-sensitive (SS/JrHsd) rats (hereafter referred to as $\mathrm{S}$ rats) and Dahl salt-resistant ( $\mathrm{SR} / \mathrm{JrHsd}$ ) rats (hereafter referred to as $\mathrm{R}$ rats). These rats were obtained from Harlan Sprague Dawley, Inc. (Indianapolis, IN) and were maintained on Purina Rat Chow 5008.

DNA fingerprint analysis. 10- $\mu \mathrm{g}$ aliquots of genomic DNA, prepared from spleen tissue by phenol/chloroform extraction, were digested overnight at $37^{\circ} \mathrm{C}$ with the restriction enzyme Alu I (New England Biolabs, Beverly, MA) and electrophoresed on a $1 \%$ agarose gel at $25 \mathrm{~V}$ for 24-48 h. After overnight transfer to a nylon membrane, 
ultraviolet fixation, and a 15-min prehybridization, the DNA was hybridized for $20 \mathrm{~min}$ at $50^{\circ} \mathrm{C}$ with an alkaline phosphatase-conjugated oligonucleotide, TGG AGG AGG GCT GGA GGA GGG C (Molecular Biosystems Inc., San Diego, CA) (8), corresponding to the consensus repeat sequence of the human myoglobin 33.6 minisatellite described by Jeffreys et al. (9). Samples were also probed with an oligonucleotide corresponding to the consensus repeat sequence of the human myoglobin 33.15 minisatellite, AGA GGT GGG CAG GTG GAG AGG TGG GCA GGT GG (9). These probes detect multiple loci scattered throughout the mammalian genome that contain tandem repeats of similar DNA sequence $(8,10)$. After washing the membrane, the DNA fingerprint patterns were developed overnight in a solution containing nitroblue tetrazolium and 5-bromo-4-chloro-3-indolyl phosphate as substrates for the alkaline phosphatase attached to the probe.

Blood pressure measurements. Each rat was briefly anesthetized with methoxyflurane, and a catheter (polyethylene No. 50) was implanted in the femoral artery with the distal end tunneled subcutaneously to an exit in the nape of the neck. The catheter was filled with a heparinized solution of $5 \%$ dextrose and plugged with a stainless steel obturator. After surgery, each rat was returned to its cage to recover from anesthesia; blood pressures were measured 4-6 $\mathrm{h}$ after stopping the anesthetic agent at a time when the animals were fully alert and roaming freely within their cages. To measure blood pressure, the femoral catheter was connected via an extension catheter to a low-volume pressure transducer (model P50; Gould Inc., Cleveland, $\mathrm{OH}$ ). The measurement of blood pressure was begun 30-60 min after connecting the catheter to the transducer. The transducer output was amplified and the electronically determined mean arterial pressure signal passed to an analogue-digital converter installed in an IBM AT microcomputer (IBM Instruments, Inc., Danbury, CT). The blood pressure signal was sampled every $15 \mathrm{~s}$ over a period of $2 \mathrm{~h}$ and the average of $\sim 480$ measurements was then calculated to yield each rat's mean arterial pressure. All studies were conducted in accordance with the guidelines of the Committee on Animal Research of the University of California San Francisco.

Renin gene typing. A Bgl II-RFLP in the rat renin gene was used as a marker to type for the SHR and LEW renin alleles (11). The RFLP appears to arise from variation in the number of tandem repeats (VNTR) of a 38-bp sequence in the first intron of the rat renin gene $(11,12)$. A nonisotopically labeled oligonucleotide complementary to the most conserved area of this tandemly repeated sequence was used to probe Southern blots of Bgl II-digested genomic DNA. The synthetic probe for the renin VNTR, AGT GCT CCC ACA GCC AGC CCA TGG G, was end-labeled with digoxegenin-labeled deoxyuridine triphosphate by a terminal transferase reaction (Genius Kit from Boehringer-Mannheim Diagnostics, Inc., Houston, TX). 10- $\mu$ g aliquots of genomic DNA, prepared from spleen tissue by phenol/chloroform extraction, were digested for $2 \mathrm{~h}$ at $37^{\circ} \mathrm{C}$ with the restriction enzyme Bgl II according to the conditions specified by the enzyme's manufacturer, Boehringer-Mannheim Diagnostics, Inc. The digested DNA samples were electrophoresed on a $1.5 \%$ agarose gel at $35 \mathrm{~V}$ for $18 \mathrm{~h}$. After Southern transfer to a nylon membrane, the DNA was fixed by ultraviolet irradiation and prehybridized for $1 \mathrm{~h}$ in blocking solution as specified in the Boehringer-Mannheim Genius Kit. The membranes were hybridized to the labeled renin probe $(50 \mathrm{ng} / \mathrm{ml}$ of hybridization buffer) at $64-65^{\circ} \mathrm{C}$ for $18 \mathrm{~h}$. After washing the membranes, the hybridized DNA was incubated with an anti-digoxegenin antibody labeled with alkaline phosphatase. Hybridized DNA was detected by an enzymatic color reaction generated by immersion of the membrane in a buffered solution containing nitroblue tetrazolium and 5-bromo4-chloro-3-indolyl phosphate.

\section{Results}

DNA fingerprints generated with either the myoglobin 33.6 or 33.15 probe were identical among rats within each parental strain (example in Fig. 1). While the apparently uniform DNA fingerprint patterns within the SHR and LEW sampled does not exclude the possibility of an undesirable degree of heterozygosity within these strains, the findings are consistent with the vendor's claim that within each strain, the rats have been brother-sister mated for multiple generations. The capacity of these minisatellite probes to reveal genetic variation within a noninbred strain of rats is unknown. With these probes, however, we have found evidence of genetic variability among Wistar-Kyoto (WKY) rats from Taconic Farms, Inc. (Germantown, NY) (8) and as a consequence, we have not used Taconic WKY rats for genetic studies.

The mean arterial pressure of the parental SHR strain was significantly greater than that of the parental Lewis strain (Fig. 2). Because these measurements were obtained in male SHRs and female LEW rats, it should be recognized that at least part of this strain difference in blood pressure may be sex-related. The blood pressure variance of the SHR strain was greater than that of the normotensive Lewis strain. In previous genetic studies, greater blood pressure variance in SHR than in inbred normotensive rats has been attributed to environment-genotype interaction (13). The mean arterial pressure of the F2 population was shifted toward that of the parental Lewis strain, with few F2 rats exhibiting hypertension as severe as that characterizing the SHR strain. However, the variance in the F2 population appeared large enough to allow for the identification of genetically defined subgroups with significantly different blood pressures.

A Southern blot depicting the RFLP in the renin gene of various rat strains is shown in Fig. 3. The oligonucleotide probe directed at the tandem repeat in the first intron allows clear distinction between the SHR and LEW strains. In SHR, the probe detects a $1.7-\mathrm{kb}$ band and in LEW rats, a 2.9-kb band; F1 rats show both bands. With this probe-enzyme combination, the $R$ rat exhibits a $1.7-\mathrm{kb}$ band, whereas the $S$ rat exhibits a $2.7-\mathrm{kb}$ band.

Data on renin genotype and blood pressure in the F2 population are presented in Fig. 4. The results for males and females have been combined as similar blood pressure/genotype results were obtained when data from males and females were analyzed separately. The frequency of renin genotypes con-

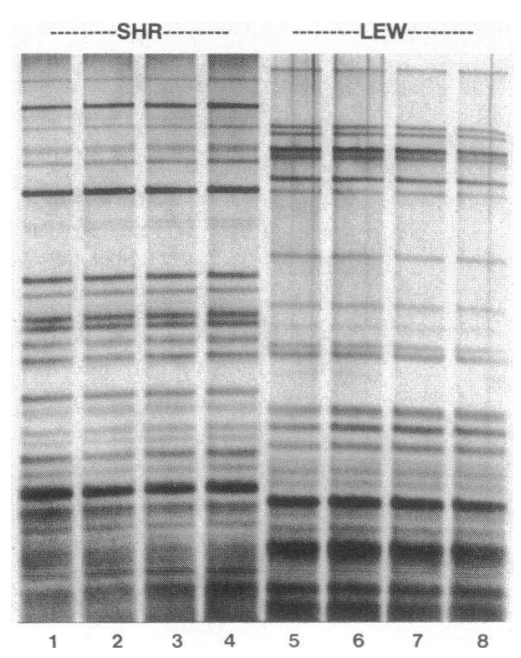

Figure 1. DNA fingerprints of inbred SHR (lanes 1-4) and LEW rats (lanes 5-8) generated with an oligonucleotide corresponding to the consensus repeat sequence of the human myoglobin 33.6 minisatellite (8-10). DNA fingerprint patterns generated with an oligonucleotide corresponding to the consensus repeat sequence of the myoglobin 33.15 minisatellite were also identical among rats within each strain (not shown) $(9$, $10)$. 


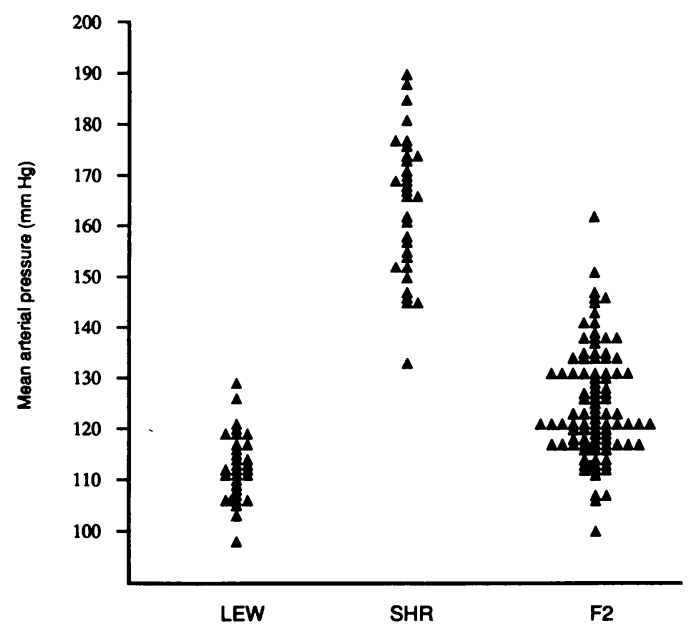

Figure 2. Mean arterial pressures in 22-26-wk-old, unanesthetized, unrestrained, LEW rats, SHR, and F2 rats. Each point represents the average of at least 400 measurements of mean arterial pressure per rat.

formed to a 1:2:1 distribution (chi-square analysis) as expected for Mendelian inheritance. Analysis of variance revealed a significant overall effect of renin genotype on blood pressure $(P$ $<0.01$ ), with the mean arterial pressure in F2 rats inheriting the SHR renin allele being greater than that in F2 rats inheriting only the Lewis renin allele. On subgroup analysis, the mean blood pressure of the heterozygotes was clearly greater than that of rats homozygous for the Lewis renin allele $(P$ $<0.001$ by one-tailed $t$ test). The blood pressure of the F2 rats homozygous for the SHR renin allele appeared greater than that of rats homozygous for the Lewis allele, although the probability value attained only borderline statistical significance $(P=0.06)$ (statistical significance was defined as $P$ $<0.017$ after the Bonferroni correction for multiple comparisons). The blood pressure of rats inheriting two SHR renin alleles was not significantly different from that of rats inheriting one SHR renin allele.

\section{Discussion}

In an F2 population derived from inbred SHR and inbred normotensive LEW rats, we have found the blood pressure in rats inheriting a single SHR renin allele to be significantly greater than that in rats inheriting only the Lewis renin allele.

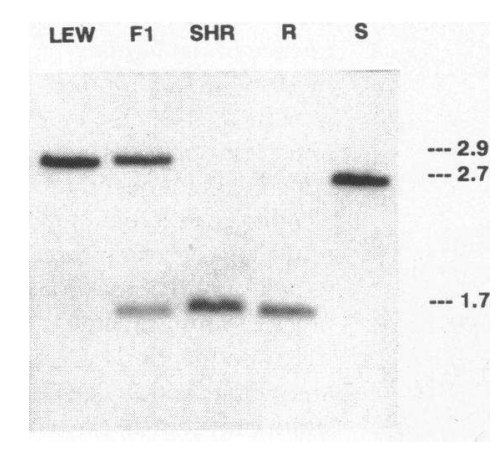

Figure 3. Southern blot of spleen genomic DNA from a LEW rat, an F1 rat (derived by crossing a SHR and a LEW rat), a SHR, an $R$ rat, and an $S$ rat. The blot was generated by probing Bgl II digested DNA with a nonisotopically labeled oligonucleotide corresponding to a tandemly repeated sequence in the first intron of the rat renin gene.

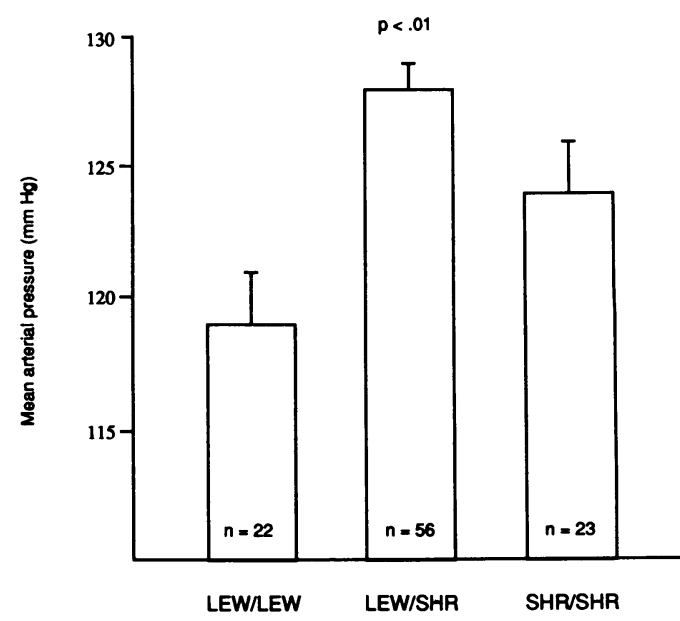

Figure 4. Bar graph of mean arterial pressures in F2 rats classifed according to renin genotype. LEW/LEW, blood pressure of rats inheriting both renin alleles from the Lewis strain; LEW/SHR, blood pressure of rats inheriting one renin allele from the Lewis strain and one renin allele from the SHR strain; SHR/SHR, blood pressure of rats inheriting both renin alleles from the SHR strain. Vertical bars and their brackets indicate group means \pm SE. The number of rats in each group is designated by the $n$ value at the base of each bar. $P$ value indicates overall statistical significance from the analysis of variance between groups.

Assuming that the alleles of the various loci that affect blood pressure are randomly segregating within the F2 population, these findings suggest that the SHR renin allele has a greater capacity to increase blood pressure than the Lewis renin allele.

In a recent study in Dahl $S$ and $R$ rats in which the amount of dietary sodium chloride provided was almost 15 times greater than that in the current experiment, Rapp and colleagues also found that a Bgl II-RFLP in the first intron of the renin gene (14) cosegregated with blood pressure (15). The blood pressure in F2 rats inheriting the renin allele of the $S$ strain was greater than that in $\mathrm{F} 2$ rats inheriting the renin allele of the $\mathbf{R}$ strain. This finding suggested that in the circumstance of a massively increased dietary intake of sodium chloride, sequence alterations in the renin gene or a closely linked gene might have the capacity to affect blood pressure. The current observations suggest that sequence alterations in the renin gene or a closely linked gene might have the capacity to affect blood pressure not only in a different genetic model of hypertension, but also in the circumstance of a near "normal" dietery intake of sodium chloride (16). Given that the renin-angiotensin-aldosterone system is a major determinant of sodium balance and vascular resistance, it would seem likely that the renin gene itself is the blood pressure regulatory gene marked by the renin RFLP.

In the study in Dahl rats in which an RFLP in the renin gene cosegregated with increased blood pressure (15), it appeared that two doses of the Dahl S renin allele conferred a greater increase in blood pressure than did a single dose of the $\mathrm{S}$ renin allele. In the current study, the blood pressure in rats inheriting two doses of the SHR renin allele was not greater than that of rats inheriting a single dose. This finding raises the possibility that under some environmental and genetic circumstances, blood pressure may scale in a nonlinear fashion with respect to the amount of renin gene product. For complex 
quantitative traits, a nonlinear relationship between phenotype and genotype is not uncommon $(17,18)$. With some genes that regulate complex quantitative traits, the phenotypic response of the heterozygotes may even lie outside the phenotypic range of the homozygotes $(17,18)$. Consideration should also be given to the possibility that another blood pressure regulatory gene is linked to the renin locus and that SHR, but not LEW rats, carry recessive alleles at this locus. Thus, in the F2 rats inheriting two SHR renin alleles, the increase in blood pressure otherwise expected to occur may have been attenuated by the co-inheritance of two recessive alleles tending to decrease blood pressure.

In Dahl rats, the Bgl II-RFLP in the renin gene stems from an insertion/deletion in the first intron which has also been proposed to involve the VNTR sequence (14). On Southern blot analysis with the renin VNTR probe, we have found that the $\mathrm{Bgl}$ II fragment of the $\mathrm{S}$ rat is distinctly different from that of the SHR and Lewis strains (Fig. 3). In contrast, the Bgl II fragment of the normotensive $R$ rat comigrates with that of the SHR (Fig. 3), suggesting that VNTR copy number is similar in these two strains. Because the SHR appears to share the same renin RFLP as the normotensive Dahl rat, one might suggest that VNTR copy number itself is not involved in the regulation of blood pressure. However, such interstrain comparisons should be interpreted with caution; in each of these strains, the final blood pressure level is determined by the interaction of multiple genes, not just the renin gene. Furthermore, although these strains exhibit similar size RFLPs, they might still differ with respect to sequences within the VNTR region or within other areas of the renin gene. It is unknown whether the renin allele in the SHR strain differs from those in the Dahl strains with respect to its capacity to increase blood pressure. For example, it is conceivable that the renin allele in the SHR, although having a greater capacity to increase blood pressure than that in the LEW rat, may have a lesser capacity to increase blood pressure than that in the Dahl S rat.

Given that multiple genes are involved in the pathogenesis of spontaneous hypertension (13), sequence variation at a single locus is not expected to account for all of the difference in blood pressure between two genetically divergent strains. It is difficult to draw conclusions regarding the extent to which a particular DNA sequence might contribute to the pathogenesis of high blood pressure: depending on the environmental circumstances and normotensive "controls" that are experimentally employed, a given allele could appear to exhibit either a large effect on blood pressure or none whatsoever. In the current study, as in the study in Dahl rats (15), only a portion of the difference in blood pressure between the high blood pressure strain and the "normal" blood pressure strain might be attributed to differences at or near the renin locus. Nevertheless, the current findings taken together with those of Rapp and co-workers (15) strongly suggest that in rats, a number of renin alleles exist with varying capacities to increase blood pressure and some may participate in the pathogenesis of hypertension. Furthermore, the capacity of different renin alleles to have different effects on blood pressure may not be limited to a unique set of environmental and genetic circumstances.

The mechanism whereby different renin alleles might have different effects on blood pressure is unknown. In SHR, it is unclear whether increased plasma renin activity is a determinant of increased blood pressure (2, 19-21). However, Samani and co-workers recently found that in 5-wk-old SHR compared with age-matched WKY rats (source unknown), renin gene expression is increased within the kidney (22). It also appears that SHRs exhibit exaggerated increases in kidney renin gene expression in response to stimuli that increase renin release, at least when compared with WKY rats from Charles River Breeding Laboratories, Inc. (23). A genetically determined increase in intrarenal renin or prorenin $(24,25)$, with or without changes in plasma renin activity, might increase blood pressure by inducing increased intrarenal formation of angiotensin II and a shift in the pressure-natriuresis curve (26). Finally, it is important to recognize that in the setting of hypertension, even a "normal" level of plasma renin activity is inappropriate (27). To the extent that the SHR provides a suitable model of "nonmodulating" hypertension $(3,4)$, the current findings raise the possibility that a structural alteration in the renin gene, or a closely linked gene, may be a pathogenetic determinant of increased blood pressure in one of the most common forms of essential hypertension in humans.

\section{Acknowledgments}

We thank J. Edman and M. Evans-Holmes for their assistance with the oligonucleotide-labeling procedure and L. Marton and R. Curtis Morris for continuing support.

This work was supported by grants from the National Institutes of Health (HL-37696 and HL-01490). Dr. T. W. Kurtz is a recipient of a Public Health Service Clinical Investigator Award from the National Heart, Lung, and Blood Institute.

\section{References}

1. Okamoto, K., and K. Aoki. 1963. Development of a strain of spontaneously hypertensive rats. Jpn. Circ. J. 27:282-293.

2. Yamori, Y. 1983. Physiopathology of the various strains of spontaneously hypertensive rats. In Hypertension, Physiopathology and Treatment. J. Genest, O. Kuchel, P. Hamet, and M. Cantin, editors. McGraw-Hill Inc., New York. 556-581.

3. Williams, G. H., L. M. Braley, and A. Menachery. 1982. Decreased adrenal responsiveness to angiotensin II: a defect present in spontaneously hypertensive rats. J. Clin. Invest. 69:31-37.

4. Guidi, E., and N. K. Hollenberg. 1987. Differential pressor and renal vascular reactivity to angiotensin II in spontaneously hypertensive and Wistar-Kyoto rats. Hypertension (Dallas). 9:591-597.

5. Shoback, D. M., G. H. Williams, T. J. Moore, R. G. Dluhy, S. Podolsky, and N. K. Hollenberg. 1983. Defect in the sodium-modulated tissue responsiveness to angiotensin II in essential hypertension. J. Clin. Invest. 72:2115-2124.

6. Lifton, R. P., P. N. Hopkins, R. R. Williams, N. K. Hollenberg, G. H. Williams, and R. G. Dluhy. 1989. Evidence for heritability of non-modulating essential hypertension. Hypertension (Dallas). 13:884-889.

7. Laragh, J. H. 1973. Vasoconstriction-volume analysis for understanding and treating hypertension: the use of renin and aldosterone profiles. Am. J. Med. 55:261-274.

8. Kurtz, T. W., M. Montano, L. Chan, and P. Kabra. 1989. Molecular evidence of genetic heterogeneity in Wistar-Kyoto rats: implications for research with the spontaneously hypertensive rat. Hypertension (Dallas). 13:188-192.

9. Jeffreys, A. J., V. Wilson, and S. L. Thein. 1985. Hypervariable "minisatellite" regions in human DNA. Nature (Lond.). 314:67-73.

10. Jeffreys, A. J. 1987. Highly variable minisatellites and DNA fingerprints. Biochem. Soc. Trans. 15:309-317.

11. Samani, N. J., W. J. Brammar, and J. D. Swales. A major 
structural abnormality in the renin gene of the spontaneously hypertensive rat. J. Hypertens. 7:249-254.

12. Fukamizu, A., K. Nishi, T. Cho, M. Saitoh, K. Nakayama, H. Ohkubo, S. Nakanishi, and K. Murakami. 1988. Structure of the rat renin gene. J. Mol. Biol. 201:443-450.

13. Tanase, H., Y. Suzuki, A. Ooshima, Y. Yamori, and K. Okamoto. 1970. Genetic analysis of blood pressure in spontaneously hypertensive rats. Jpn. Circ. J. 34:1197-1212.

14. Wang, S. M., and J. P. Rapp. 1989. Structural differences in the renin gene of Dahl salt-sensitive and salt-resistant rats. Mol. Endocrinol. 3:288-294.

15. Rapp, J. P., S.-M. Wang, and H. Dene. 1989. A genetic polymorphism in the renin gene of Dahl rats cosegregates with blood pressure. Science (Wash. DC). 243:542-544.

16. Kurtz, T. W., and R. C. Morris. 1985. Hypertension in the recently weaned Dahl salt-sensitive rat despite a diet deficient in sodium chloride. Science (Wash. DC). 230:808-810.

17. Lewontin, R. C. 1981 . Quantitative genetics. In Introduction to Genetic Analysis. D. T. Suzuki, A. J. F. Griffiths, and R. C. Lewontin, editors. W. H. Freeman and Co., San Francisco. 807-848.

18. Pooni, H. S., and J. L. Jinks. 1980. Non-linear genotype $\times$ environment interactions. II. Statistical models and genetic control. Heredity. 45:389-400.

19. Kawabe, K., T. X. Watanabe, K. Shiono, and H. Sokabe. 1978. Influence on blood pressure of renal isografts between spontaneously hypertensive and normotensive rats, utilizing the F1 hybrids. Jpn. Heart J. 19:886-894.
20. Bagby, S. P., W. J. McDonald, and R. D. Mass. 1979. Serial renin-angiotensin studies in spontaneously hypertensive and WistarKyoto normotensive rats. Hypertension (Dallas). 1:347-354.

21. Harrap, S. B., and A. E. Doyle. 1988. Genetic cosegregation of renal hemodynamics and blood pressure in the spontaneously hypertensive rat. Clin. Sci. (Lond). 74:63-69.

22. Samani, N. J., W. J. Brammar, and J. D. Swales. 1988. Renal and extra-renal renin gene expression-effects of salt intake, hypertension, and genetic background. J. Hypertens. 6:940A. (Abstr.)

23. Kitami, Y., K. Hiwada, and T. Kokubu. 1989. Kidney renin gene expression in spontaneously hypertensive rats. J. Hypertens. 7:727-731.

24. Dzau, V. J., D. W. Burt, and R. E. Pratt. 1988. Molecular biology of the renin-angiotensin system. Am. J. Physiol. 255:F563F573.

25. Sealy, J. E., and S. Rubattu. 1989. Prorenin and renin as separate mediators of tissue and circulating systems. Am. J. Hypertens. 2:358-366.

26. Guyton, A. C. 1987. Renal function curve-a key to understanding the pathogenesis of hypertension. Hypertension (Dallas). 10:1-6.

27. Sealy, J. E., J. D. Blumenfeld, G. M. Bell, M. S. Pecker, S. C. Sommers, and J. H. Laragh. 1988. On the renal basis for essential hypertension: nephron heterogeneity with discordant renin secretion and sodium excretion causing a hypertensive vasoconstriction-volume relationship. J. Hypertens. 6:763-777. 